\title{
Cost-effectiveness analysis of adjuvant treatment for resected pancreatic cancer in China based on the ESPAC-4 trial
}

This article was published in the following Dove Press journal: Cancer Management and Research

\author{
Jiaxing Huang ${ }^{1,2, *}$ \\ Weiting Liao ${ }^{1,2, *}$ \\ Jing Zhou',2 \\ Pengfei Zhang ${ }^{1,2}$ \\ Feng Wen ${ }^{1,2}$ \\ Xinyuan Wang ${ }^{1,2}$ \\ Mengxi Zhang ${ }^{1,2}$ \\ Kexun Zhou ${ }^{1,2}$ \\ Qiuji Wu ${ }^{1,2}$ \\ Qiu $\mathrm{Li}^{1,2}$
}

'Department of Medical Oncology, Cancer Center, West China Hospital, Sichuan University, Chengdu 6I004I.

China; ${ }^{2}$ West China Biomedical Big

Data Center, Sichuan University,

Chengdu 61004I, China

*These authors contributed equally to this work
Correspondence: Qiu Li

Department of Medical Oncology,

Cancer Center,

West China Hospital, Sichuan University.

No. 37, Guo Xue Xiang, Chengdu,

Sichuan, 61004I, China

Tel +86 2885423262

Fax +862885423609

Email fbqiu9@।63.com
Background: The effectiveness of gemcitabine plus capecitabine compared with gemcitabine monotherapy for resected pancreatic cancer has been evaluated in the ESPAC-4 trial. We aimed to assess the cost-effectiveness of these adjuvant regimens on resected pancreatic cancer.

Methods: A Markov model was established to simulate the disease process of resected pancreatic cancer (relapse-free survival, progressive disease, and death). The efficacy and toxicity profiles were collected from the ESPAC-4 trial. Transition probabilities were calculated based on survival in each group. Cost data were calculated from the perspective of the Chinese healthcare payer. The primary endpoint in the analysis was the incremental cost-effectiveness ratio (ICER), and model uncertainties were explored by one-way sensitivity analysis and probabilistic sensitivity analysis.

Results: Our results demonstrated that gemcitabine monotherapy cost $\$ 36,028.45$ and yielded a survival of 1.02 quality-adjusted life year (QALY), while gemcitabine plus capecitabine cost $\$ 46,095.05$ and yielded a survival of 1.23 QALY. Therefore, the incremental cost-effectiveness ratio of gemcitabine plus capecitabine vs gemcitabine monotherapy was $\$ 45,191.23$ which surpassed the willingness-to-pay threshold of \$29,291.42 per QALY in China.

Conclusion: The gemcitabine monotherapy regimen is more cost-effective compared with gemcitabine plus capecitabine regimen for the patients with postoperative pancreatic cancer from the Chinese societal perspective.

Keywords: cost-effectiveness, Markov model, gemcitabine, capecitabine, resected pancreatic cancer

\section{Introduction}

Pancreatic cancer is a common and highly fatal cancer, with a poor prognosis. ${ }^{1}$ In pancreatic cancer patients a 5 -year survival rate is only $8 \%$, even though there has been a gradual increase in survival for most cancers over the decades. ${ }^{2}$ More than $80 \%$ of patients with pancreatic cancer are asymptomatic and exhibit unresectable advanced pancreatic cancer at diagnosis. ${ }^{3}$ Only $20 \%$ of patients are eligible for initial resection. ${ }^{4}$ However, after radical resection, most patients will experience recurrence within 2 years. ${ }^{5,6}$ Surgical resection with adjuvant chemotherapy, with either 5-fluorouracil plus folinic acid or gemcitabine, has increased the 5-year survival rate to $\sim 20 \% .^{7-11}$ Recently, several studies indicated that adjuvant chemotherapy was an effective means for resected pancreatic patients to obtain long-term survival and it is steadily accepted as the established standard of care. ${ }^{8,10,12-16}$

Gemcitabine had been associated with significant improvement in disease-free survival (DFS) and overall survival (OS) in postoperative pancreatic patients com- 
pared with placebo cohort (median DFS: 13.4 months vs 6.9 months; median OS: 22.8 months vs 20.2 months). ${ }^{5,17}$ The combination of gemcitabine and capecitabine has synergistic effect on thymidylate synthase involved in normal DNA synthesis. ${ }^{18}$ Moreover, previous clinical trials have demonstrated this combination produced a better tumor response with well tolerated adverse effects compared with monotherapy in patients with advanced pancreatic cancer. ${ }^{19,20}$

The European study group for pancreatic cancer (ESPACIV) trial was performed to evaluate efficacy and safety of gemcitabine plus capecitabine compared with gemcitabine monotherapy for postoperative pancreatic cancer. The results revealed that the gemcitabine plus capecitabine regimen significantly improved median overall survival (OS) and median relapse-free survival (RFS) compared with gemcitabine (28.0 months vs 25.5 months, $P=0.032 ; 13.9$ months vs 13.1 months, $P=0.082$ ). Grade 3-4 adverse events, neutropenia, white blood cell count decrease, and hand-foot syndrome were frequently reported in the gemcitabine plus capecitabine cohort $(38 \%, 10 \%, 7 \%)$, whereas neutropenia, white blood cell count decrease, infection and infestations were significantly greater in the gemcitabine monotherapy cohort $(24 \%$, $8 \%, 7 \%$ ). Thus, the combination of the gemcitabine and capecitabine regimen seemed to be a more effective option for the treatment of resected pancreatic cancer. ${ }^{18}$

Even though gemcitabine plus capecitabine regimen have proven to have a better clinical response when compared with gemcitabine monotherapy, they have not been directly compared in terms of being cost effective. Taking costeffectiveness into consideration is crucial for clinicians to make an optimal decision, as well as from a social perspective. Herein, we performed a Markov model to evaluate the cost-effectiveness of gemcitabine plus capecitabine compared with gemcitabine monotherapy for resected pancreatic cancer from the perspective of a Chinese society.

\section{Materials and methods}

\section{Patients and regimens}

The clinical data for this model was derived from the ESPACIV trial, a multicenter, open-label, randomized, phase III trial conducted in 92 hospitals in England, Scotland, Wales, Germany, France, and Sweden. ${ }^{18}$ The inclusion criteria were patients aged 18 years or older who had undergone complete resection for pancreatic cancer. ${ }^{18}$ The eligible patients were randomly assigned within 12 weeks of resection to receive 6 cycles of either $1000 \mathrm{mg} / \mathrm{m}^{2}$ gemcitabine alone, administered once a week for 3 of every 4 weeks cycle, or with $1660 \mathrm{mg} / \mathrm{m}^{2}$ oral capecitabine administered for 21 days followed by a 7 day rest per cycle. ${ }^{18}$ Laboratory tests, clinical symptoms, tumor markers, chest radiographs and abdominal CT were assessed based on the protocol of ESPAC-4 trial. ${ }^{18}$ The median RFS was 13.9 months in gemcitabine plus capecitabine cohort, and 13.1 months in gemcitabine monotherapy cohort The median overall survival (OS) of gemcitabine plus capecitabine and gemcitabine monotherapy was 28.0 and 25.5 months, respectively. ${ }^{18}$ The other primary input clinical efficacy parameters are shown in (Table 1).

\section{Model structure}

A Markov model was performed with TreeAge Pro 2011 (TreeAge Software, Inc., Williamstown, MA, USA) to simulate the disease process of resected pancreatic cancer and compare the cost-effectiveness of 2 strategies based on the ESPAC-4 trials. The decision model structure comprised 3 mutually exclusive states including RFS, progressive disease (PD), and death (Figure 1). The patients could shift to a different state at the end of each cycle in the Markov model, according to the transition probabilities calculated by the 5-year RFS rate; and 5-year OS rate (Table 1), and costs and benefits were discounted to present values at $3 \%$ for 1 year. ${ }^{21}$ The model cycle length was 1 month, and the time horizon was 10 years. Monthly transition probabilities of health states were calculated by the following formula: $r=\left[1-\ln \left(1-\mathrm{P}_{1}\right)\right] / \mathrm{t}$, $\mathrm{P}_{2}=1$-exp(-ru), $\mathrm{r}$ : instantaneous rate; $\mathrm{P}_{1}$ : cumulative probability at time $t$ (5 years), $u$ : model cycle length, $\mathrm{P}_{2}$ : Monthly transition probabilities..$^{22,23}$

\section{Cost estimate}

Total costs in our analysis consisted of direct medical costs and societal costs. Cost of drugs and tests were derived from the 2018 fee standards of West China Hospital, Sichuan University. The median relative dose intensity (RDI) of the RFS state drugs in gemcitabine group and gemcitabine plus capecitabine were $83 \%, 78 \%$, respectively. ${ }^{18}$ Direct medical costs included drugs, tests, inpatient fees and treatments for grade 3-4 AEs. The grade 3-4 AEs rates sourced from the trials were used to calculate the AE-related costs (Table 1), whereas societal costs consisted of travel fees and time costs (absenteeism fees), and travel costs were assessed at $\$ 10.20$ per patient each trip to the hospital in Sichuan, China, in $2016 .{ }^{24}$ Time costs were estimated at $\$ 35.73$ per day based on the average monthly salary in China in $2017 .{ }^{24}$ Travel costs and time costs were derived from the average length inpatient hospitalization of 2 times per month, 3 days each time and outpatient visits of 2 times per month. For the cost of PD, in patients chiefly treated with platinum-based chemotherapy 
Table I Clinical efficacy and adverse events of gemcitabine plus capecitabine and gemcitabine monotherapy

\begin{tabular}{|c|c|c|c|}
\hline \multirow[t]{2}{*}{ Variable } & \multicolumn{3}{|l|}{ Base-case value } \\
\hline & GEM & GEMCAP & Reference \\
\hline \multicolumn{4}{|l|}{ Clinical efficacy, months $(95 \% \mathrm{Cl})$} \\
\hline Median OS (m) & $25.5(22.7-27.9)$ & $28.0(23.5-31.5)$ & 18 \\
\hline Median RFS (m) & I3.I (II.6-15.3) & $13.9(12.1-16.6)$ & 18 \\
\hline 5-year OS rate & $16.3 \%(10.2-23.7)$ & $28.8 \%(22.9-35.2)$ & 18 \\
\hline 5-year RFS & $11.9 \%(7.8-16.9)$ & $18.6 \%(\mid 3.8-24.0)$ & 18 \\
\hline \multicolumn{4}{|l|}{ Probability of grade $3 / 4$ AEs, $\%$} \\
\hline Anemia & 4 & 2 & 18 \\
\hline Diarrhea & 2 & 5 & 18 \\
\hline Fatigue & 5 & 6 & 18 \\
\hline Fever & 2 & 2 & 18 \\
\hline Infection & 7 & 3 & 18 \\
\hline Lymphocyte count decreased & 3 & 3 & 18 \\
\hline Neutropenia & 24 & 38 & 18 \\
\hline Hand-foot syndrome & 0 & 7 & 18 \\
\hline Thrombocytopenia & 2 & 2 & 18 \\
\hline Thromboembolic events & 2 & 2 & 18 \\
\hline White blood cell count decreased & 8 & 10 & 18 \\
\hline Acute kidney injury & I & 0 & 18 \\
\hline
\end{tabular}

Abbreviations: AEs, adverse events; GEM, gemcitabine; GEMCAP, gemcitabine plus capecitabine; OS, overall survival; RFS, relapse-free survival.

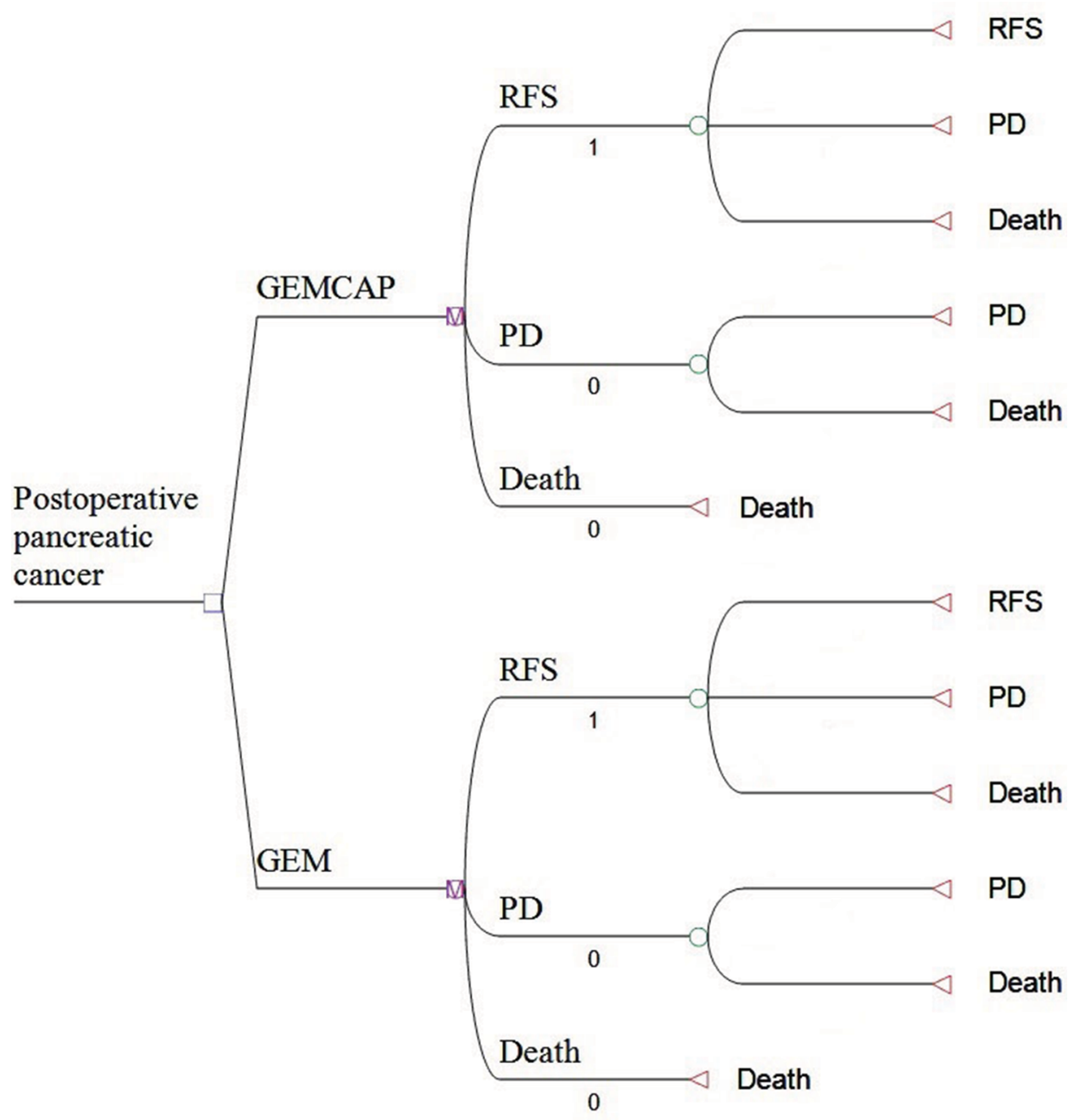

Figure I Markov model for postoperative pancreatic cancer.

Notes: Markov model for resected pancreatic cancer. A Markov model comprising 3 health states (relapse-free survival, PD and death) was built. Abbreviations: GEM, gemcitabine; GEMCAP, gemcitabine plus capecitabine, PD, progressive disease; RFS, relapse-free survival. 
regimens, a weighted cost based on FOLFIRINOX (5-FU, leucovorin, oxaliplatin, irinotecan), GEM-N (gemcitabine, nab-paclitaxel) was assumed per cycle. ${ }^{25,26}$ The RDIs for these treatments were assumed to be $80 \% .{ }^{27}$ All costs were converted to USD, at an exchange rate of $\$ 1=$ RMB 6.33, in March 2018.

\section{Effectiveness estimates}

Treatment effectiveness was estimated by QALYs. Utility scores of Markov states were based on the previous studies, with 0.85 for RFS state and 0.73 for PD state. ${ }^{28,29}$

\section{Sensitivity analysis}

One-way sensitivity analysis was performed to investigate the impact of variables on the analysis model by varying the necessary parameters within a range of $\pm 30 \%$. As for probabilistic sensitivity analysis, a Monte Carlo simulation of 1,000 iterations was developed to assess the uncertainty strategies, and the results were presented as cost-effectiveness acceptability curves. According to WHO guidelines, the willingness to pay (WTP) threshold value was 3 times Gross Domestic Product per Capita (GDP) of China in 2017, which was $\$ 25,840.88$ / QALY, ie $\$ 2,153.40$ per quality-adjusted life month. ${ }^{30}$

\section{Results}

\section{Costs outcomes}

The estimated monthly costs of the 2 treatments are briefly presented in (Table 2).

As for the cost for RFS state, the greatest cost was RDIadjusted drugs (\$1,237.01 for gemcitabine and $\$ 1,726.15$ for gemcitabine plus capecitabine). The inpatient fees, test costs and total societal costs were the same in these 2 groups. Moreover, the grade 3-4 adverse effects related to cost were similar (\$41.91 for gemcitabine and \$55.67 for gemcitabine plus capecitabine). As for the cost of PD state, the total cost was $\$ 2,643.56$ for both treatment groups. After running the Markov model to the estimated time horizon, the cumulative costs were $\$ 36,028.45$ for the gemcitabine group, which was significantly lower than that of $\$ 46,095.05$ for the gemcitabine plus capecitabine group (Table 3 ).

\section{Cost-effectiveness}

As shown in Table 3, according to the cost analysis and effectiveness analysis described previously, the gemcitabine monotherapy was cheaper, with a cost of \$35,322.01/QALY compared with $\$ 45,191.23 /$ QALY for the combination of gemcitabine and capecitabine. Gemcitabine plus capecitabine group provided an incremental 0.21 QALYs at an incremental
Table 2 Cost and utility scores of gemcitabine plus capecitabine and gemcitabine monotherapy

\begin{tabular}{lll}
\hline Variable & \multicolumn{2}{l}{ Base-case value } \\
\cline { 2 - 3 } & GEM & GEMCAP \\
\hline Costs for RFS state (USD/month) & & \\
Chemotherapy drugs & $1,490.37$ & $2,213.01$ \\
RDI-adjusted drugs & $1,237.01$ & $1,726.15$ \\
Inpatient fees & 140.23 & 140.23 \\
Grade 3-4 AEs & 41.91 & 55.67 \\
Test & 148.13 & 148.13 \\
Societal costs (USD/month) & & \\
Time & 214.21 & 214.21 \\
Travel & 40.86 & 40.86 \\
Total societal costs & 255.07 & 255.07 \\
Total & $1,822.35$ & $2,325.25$ \\
Total costs for PD state (USD/month) & & \\
Weighted average drugs for PD state & $2,764.14$ & $2,764.14$ \\
RDI-adjusted drugs & $2,211.31$ & $2,211.31$ \\
Additional cost for PD state & 432.25 & 432.25 \\
Total & $2,643.56$ & $2,643.56$ \\
Utility scores & & \\
Utility for RFS state & 0.85 & 0.85 \\
Utility for PD state & 0.73 & 0.73 \\
\hline A & &
\end{tabular}

Abbreviations: AE, adverse event; GEM, gemcitabine; GEMCAP, gemcitabine plus capecitabine; PD, progressive disease; RDI, relative dose intensity; RFS, relapse-free survival.

Table 3 Results of cost-effectiveness analysis of gemcitabine plus capecitabine and gemcitabine monotherapy

\begin{tabular}{lll}
\hline Result & \multicolumn{2}{l}{ Regimen } \\
\cline { 2 - 3 } & GEM & GEMCAP \\
\hline Costs (USD) & $27,460.90$ & $33,539.46$ \\
Costs for the RFS state & $8,567.55$ & $12,555.59$ \\
Costs for the PD state & $36,028.45$ & $46,095.05$ \\
Total & & \\
Effectiveness QALYs (USD) & 0.73 & 0.90 \\
Effectiveness for the PFS state & 0.29 & 0.33 \\
Effectiveness for the PD state & 1.02 & 1.23 \\
Total effectiveness & $35,322.01$ & $45,191.23$ \\
C/E ratio (USD\$/QALY) & $47,936.19$ & \\
ICER for GEMCAP VS. (GEM USD/QALY) & \\
\hline
\end{tabular}

Abbreviations: $A E$, adverse event; $C / E$, cost/effectiveness ratio; $G E M$, gemcitabine; GEMCAP, gemcitabine plus capecitabine; ICER, incremental cost-effectiveness ratio; $\mathrm{PD}$, progressive disease; RDI, relative dose intensity; RFS, relapse-free survival; QALY, quality-adjusted life year.

cost of $\$ 10,066.60$, compared with the gemcitabine group, resulting in the ICER of $\$ 47,936.19 / \mathrm{QALY}$, which exceeded the WTP threshold of $\$ 25,840.88 /$ QALY.

\section{Sensitivity analysis}

The one-way sensitivity analyses are displayed in the tornado diagram (Figure 2). The cost of the PD state in gemcitabine plus capecitabine cohort and the cost of the PD state in the gemcitabine cohort played a vital role in our study. When cost 


\section{Tornado Analysis (ICER)}

GEMCAP cost value of PD

GEM cost value of PD

GEMCAP cost value of RFS

GEM cost value of RFS

Utility score of RFS

GEM cost value

Utility score of PD

GEMCAP p of RFS to PD

Inpatient fee of GEMCAP group

Inpatient fee of GEM group

Cost of 3/4 AEs for GEMCAP group

Cost of 3/4 AEs for GEM group

Cost of tests

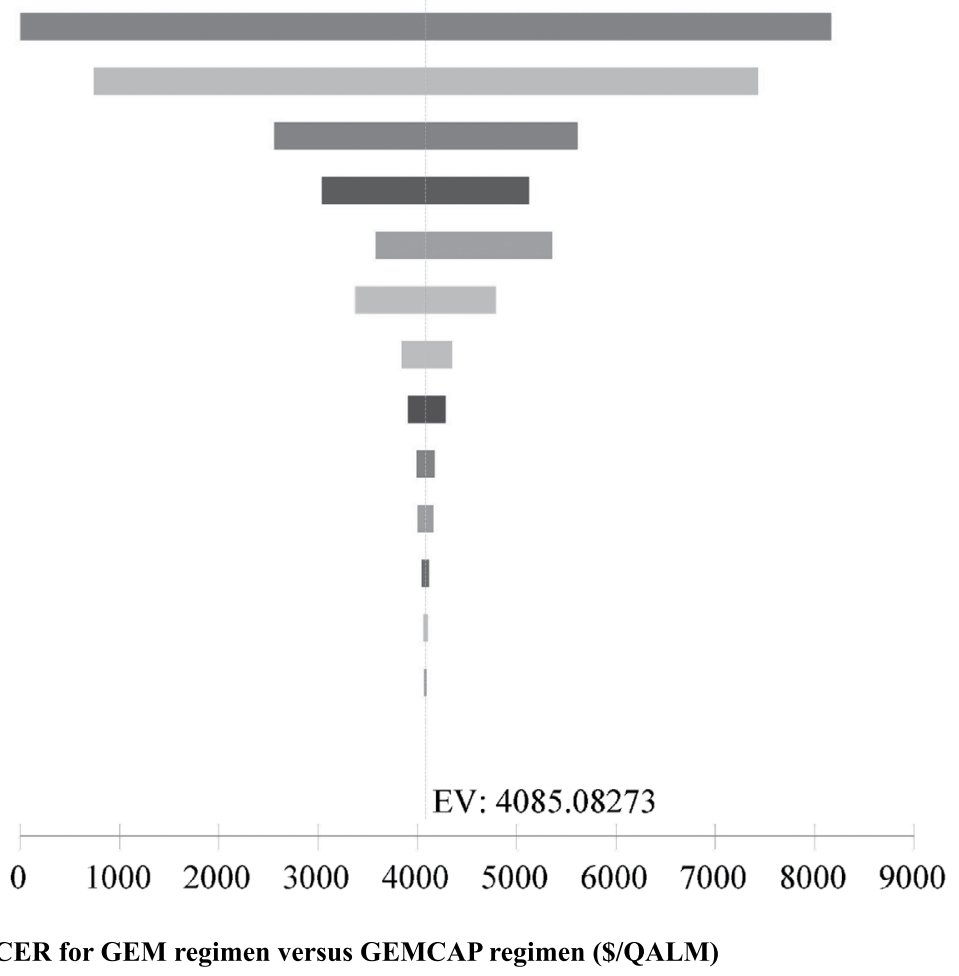

Figure 2 Tornado diagram of one-way sensitivity analyses.

Notes: Tornado diagrams show the influence of factors on the Markov model. The factors are listed in descending order of the influence on ICER with variation of factor values.

Abbreviations: AE, adverse event; GEM, gemcitabine; GEMCAP, gemcitabine plus capecitabine; ICER, incremental cost-effectiveness ratio; PD, progressive disease; $P$, transition probability; QALM, quality-adjusted life month; RFS, relapse-free survival; EV, expected value.

of the PD state in the gemcitabine plus capecitabine cohort varied from $\$ 1,850.49$ to $\$ 3,436.63$, the ICER increased from $\$ 29,295.30$ to $\$ 45,656.02$ per QALY. If the cost of PD state in gemcitabine group changed from $\$ 1,850.49$ to $\$ 3,436.63$, the ICER rose from $\$ 22,593.65$ to $\$ 35,989.21$ per QALY. Nevertheless, the cost of test and cost of grade 3-4 AEs in these 2 strategies had a slight impact on the model. The result of the Monte Carlo simulation of 1,000 patients showed that the mean cost and effectiveness gained were: $\$ 46,300.77 \pm 741.49$ and 1.23 \pm 0.02 QALY for gemcitabine plus capecitabine group, while $\$ 36,243.69 \pm 652.05$ and 1.03 \pm 0.02 QALY for gemcitabine group. The probabilistic sensitivity analysis indicated nearly $100 \%$ probability of gemcitabine and $0 \%$ probability of gemcitabine plus capecitabine being a costeffective strategy, as the WTP value was \$2,153.40/QALM. (Figure 3)

\section{Discussion}

Pancreatic cancer is a seriously lethal disease, and mortality rate closely coincides with incidence. ${ }^{1}$ After resection, chemotherapy with fluorouracil or gemcitabine significantly prolongs OS and reduces the incidence of relapse. ${ }^{6}$ However, a significant burden is placed on patients during the adjuvant therapy process for resected pancreatic cancer. An economic assessment of postoperative adjuvant regimens is vital to keep the balance between clinical benefits and health care cost, especially in developing countries such as resource-limited China. ${ }^{31}$ Therefore, we established a Chinese cost-effective analysis of gemcitabine plus capecitabine vs gemcitabine alone for resected pancreatic cancer, which is the first analysis of postoperative pancreatic cancer adjuvant strategies from the efficacy and cost-effectiveness perspective.

According to our analysis, gemcitabine plus capecitabine cohort cost $\$ 2,325.25$ per month which was higher than gemcitabine alone $\$ 1,822.35$ for the RFS state. The chemotherapy drugs, test, and inpatient fees costs contributed most to the total costs of different treatment groups. Our one-way sensitivity analyses indicated that the key driver of the ICER of gemcitabine plus gemcitabine vs gemcitabine alone was the cost of the PD state in both cohorts. Gemcitabine plus capecitabine group offered an incremental 0.21 QALY at an incremental cost of $\$ 10,066.60$, compared with the gemcitabine group, 


\section{CE Acceptability Curve}

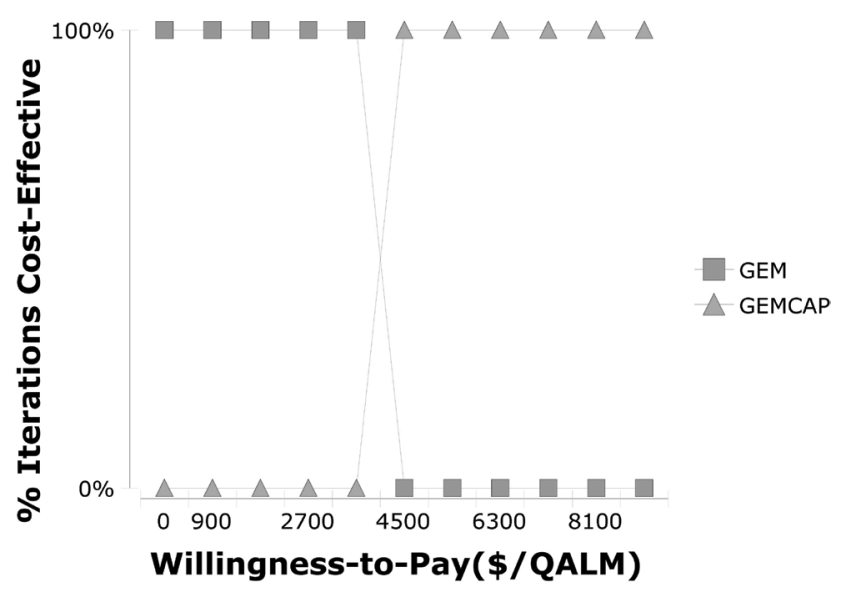

Figure 3 Cost-effectiveness acceptability curves.

Notes: The cost-effectiveness acceptability curves reflected the results of probabilistic sensitivity analysis by estimating probabilities of different treatments being considered as optimal strategies at different WTP thresholds.

Abbreviations: CE, cost effectiveness; GEM, gemcitabine; GEMCAP, gemcitabine plus capecitabine; QALM, quality adjusted life month; WTP, willingness to pay.

resulting in the ICER of $\$ 47,936.19 /$ QALY. The WTP threshold is $\$ 25,840.88 / \mathrm{QALY}$ in our model, which is triple the per capita gross domestic product of China. ${ }^{31}$ In other words, the ICER of gemcitabine plus capecitabine vs gemcitabine monotherapy dramatically surpassed the general WTP threshold in China, even though the gemcitabine plus capecitabine regimen showed better clinical response in ESPAC-4 trials. Thus, gemcitabine plus capecitabine is not an optimal costeffective regimen for postoperative pancreatic cancer from Chinese social perspective.

So far, there has been no economic evaluation for resected pancreatic cancer to compare the standard adjuvant treatment, except in metastatic background. A study reported that paclitaxel ablumin plus gemcitabine regimen offered more 0.154 QALYs and $€ 7082.68$ than gemcitabine alone regimen. Incremental cost-utility ratio $(€ 46,021.58)$ is lower than the informal threshold value of $€ 87,330$ adopted by the Italian Medicines Agency (INHS) during 2010-2013 for reimbursing oncological drugs, which means that albuminbound paclitaxel plus gemcitabine can be considered a costeffective regimen for metastatic pancreatic cancer by the Italian Medicines Agency. ${ }^{32}$ Moreover, a pharmacological evaluation compared cost-effectiveness of gemcitabine, gemcitabine plus 5-fluorouracil, gemcitabine plus capecitabine, gemcitabine plus cisplatin, gemcitabine plus oxaliplatin, gemcitabine plus erlotinib, gemcitabine plus nab-paclitaxel, and FOLFIRINOX in the treatment of advanced pancreatic cancer, the result demonstrated that FOLFIRINOX would be the most optimal treatment for advanced pancreatic cancer as the WTP threshold of $\$ 50,000$ per QALY from a Canadian public health payer threshold, and the analysis also revealed that the most cost-effective regimen relies on the societal WTP threshold..$^{33}$ Our previous study showed that S-1 regimen could provide the maximum societal benefits and sustainable maintenance of the national healthcare sector than gemcitabine alone or regimen combined with gemcitabine plus S-1 in advanced pancreatic cancer. ${ }^{34}$

Gemcitabine plus capecitabine and gemcitabine alone both maintain a manageable toxicity profile. In terms of the grade 3-4 adverse events, neutropenia, white blood cell count decreased, and hand-foot syndrome occurred more frequently in the gemcitabine plus capecitabine cohort $(38 \%, 10 \%$, $7 \%$ ), whereas neutropenia, white blood cell count decreased, infection and infestations were significantly greater in the gemcitabine monotherapy cohort $(24 \%, 8 \%, 7 \%)$. Based on our analysis, the AEs related costs were $\$ 41.91$ and $\$ 55.67$ per month in gemcitabine plus capecitabine group and gemcitabine alone, respectively. And the result revealed that the AE-related costs had a minor impact on the ICER of gemcitabine plus capecitabine vs gemcitabine alone.

There were several limitations in this cost-effective analysis. The Chinese cost-effectiveness analysis developed was based on data from the ESPAC-4 trial rather than collecting data from a clinical practice. However, previous studies have discussed the suitability of using foreign clinical data with local population and concluded that the influence of such differences between local individuals and other countries' populations on event rates should be accepted. ${ }^{35,36}$ Additionally, we established a sensitivity analysis to create a widely adaptable cost for a local area. Moreover, due to a lack of detailed information about quality of life, the utility value in our model was derived from published literature.

\section{Conclusion}

Overall, the current study was the first study to compare an adjuvant chemotherapy regimen in resected pancreatic cancer from a cost-effectiveness perspective. The result demonstrated that the gemcitabine monotherapy regimen is more cost-effective when compared with the gemcitabine plus capecitabine regimen for patients with postoperative pancreatic cancer from the Chinese societal perspective. Our analysis would contribute in aiding clinicians in making optimal decision for the treatment of resected pancreatic cancer patients.

\section{Acknowledgment}

The authors thank Prof David W Hutton from the department of Health Management and Policy, University of Michigan for his fruitful discussions on the study. This work was supported 
by the National Natural Science Foundation of China (No. 81572988) and Science \& Technology Department of Sichuan Province Funding Project (No. 2016FZ0108, 18ZDYF1981).

\section{Disclosure}

The authors report no conflicts of interest in this work.

\section{References}

1. Kamisawa T, Wood LD, Itoi T, Takaori K. Pancreatic cancer. Lancet. 2016;388(10039):73-85.

2. Sato-Dahlman M, Wirth K, Yamamoto M. Role of Gene Therapy in Pancreatic Cancer-A Review. Cancers. 2018;10(4):103.

3. Siegel RL, Miller KD, Jemal A. Cancer Statistics, 2017. CA Cancer J Clin. 20172017;67(1):7-30.

4. Gillen S, Schuster T, Meyer Zum Büschenfelde C, Friess H, Kleeff J. Preoperative/neoadjuvant therapy in pancreatic cancer: a systematic review and meta-analysis of response and resection percentages. PLOS Med. 2010;7(4):e1000267.

5. Oettle H, Neuhaus P, Hochhaus A, et al. Adjuvant Chemotherapy With Gemcitabine and Long-term Outcomes Among Patients With Resected Pancreatic Cancer. JAMA. 2013;310(14):1473.

6. Liao W-C, Chien K-L, Lin Y-L, et al. Adjuvant treatments for resected pancreatic adenocarcinoma: a systematic review and network metaanalysis. Lancet Oncol. 2013;14(11):1095-1103.

7. Neoptolemos J. ESPAC-1: A Europian randomized controlled study of adjuvant chemoradiation and chemotherapy in resectable pancreatic cancer. Lancet. 2001;358:1576-1585.

8. Neoptolemos JP, Stocken DD, Friess H, et al. A randomized trial of chemoradiotherapy and chemotherapy after resection of pancreatic cancer. N Engl J Med. 2004;350(12):1200-1210.

9. Oettle H, Neuhaus P, Hochhaus A, et al. Adjuvant chemotherapy with gemcitabine and long-term outcomes among patients with resected pancreatic cancer: the CONKO-001 randomized trial. JAMA 2013;310(14):1473-1481.

10. Neoptolemos JP, Stocken DD, Smith CT, et al. Adjuvant 5-fluorouracil and folinic acid vs observation for pancreatic cancer: composite data from the ESPAC-1 and -3(v1) trials. Br J Cancer. 2009;100(2): 246-250.

11. Valle JW, Palmer D, Jackson R, et al. Optimal duration and timing of adjuvant chemotherapy after definitive surgery for ductal adenocarcinoma of the pancreas: ongoing lessons from the ESPAC-3 study. J Clin Oncol. 2014;32(6):504-512.

12. Neoptolemos JP, Stocken DD, Bassi C, et al. Adjuvant chemotherapy with fluorouracil plus folinic acid vs gemcitabine following pancreatic cancer resection: a randomized controlled trial. JAMA. 2010;304(10):1073-1081.

13. Neoptolemos JP, Moore MJ, Cox TF, et al. Effect of adjuvant chemotherapy with fluorouracil plus folinic acid or gemcitabine vs observation on survival in patients with resected periampullary adenocarcinoma: the ESPAC-3 periampullary cancer randomized trial. JAMA. 2012;308(2):147-156.

14. Ueno H, Kosuge T, Matsuyama Y, et al. A randomised phase III trial comparing gemcitabine with surgery-only in patients with resected pancreatic cancer: Japanese Study Group of Adjuvant Therapy for Pancreatic Cancer. Br J Cancer. 2009;101(6):908-915.

15. Liao WC, Chien KL, Lin YL, et al. Adjuvant treatments for resected pancreatic adenocarcinoma: a systematic review and network metaanalysis. Lancet Oncol. 2013;14(11):1095-1103.

16. Neoptolemos JP, Cox TF. Bayesian analysis unravels pancreas-cancer adjuvant therapy. Lancet Oncol. 2013;14(11):1034-1035.

17. Oettle H, Post S, Neuhaus $P$, et al. Adjuvant chemotherapy with gemcitabine vs observation in patients undergoing curative-intent resection of pancreatic cancer: a randomized controlled trial. JAMA. 2007;297(3):267-277.
18. Neoptolemos JP, Palmer DH, Ghaneh P, et al. Comparison of adjuvant gemcitabine and capecitabine with gemcitabine monotherapy in patients with resected pancreatic cancer (ESPAC-4): a multicentre, open-label, randomised, phase 3 trial. Lancet. 2017;389(10073):1011-1024.

19. Cunningham D, Chau I, Stocken DD, et al. Phase III randomized comparison of gemcitabine versus gemcitabine plus capecitabine in patients with advanced pancreatic cancer. J Clin Oncol. 2009;27(33):5513-5518.

20. Sultana A, Smith CT, Cunningham D, Starling N, Neoptolemos JP, Ghaneh P. Meta-analyses of chemotherapy for locally advanced and metastatic pancreatic cancer. J Clin Oncol. 2007;25(18):2607-2615.

21. Gold M. Panel on cost-effectiveness in health and medicine. Med Care. 1996;34(Suppl 12):197-199.

22. Caldwell D. Decision Modelling for Health Economic Evaluation. A Briggs, M Sculpher, K Claxton. Int J Epidemiol. 2007;36(2):476-477.

23. Aalabaf-Sabaghi M. Book review of Decision Modelling For Health Economic Evaluation. A Briggs, M Sculpher, K Claxton. J Epidemiol Commun Health. 2007;61(9):839.

24. Chen HD, Zhou J, Wen F, et al. Cost-effectiveness analysis of apatinib treatment for chemotherapy-refractory advanced gastric cancer. J Cancer Res Clin Oncol. 2017;143(2):361-368.

25. Conroy T, Desseigne F, Ychou M, et al. FOLFIRINOX versus gemcitabine for metastatic pancreatic cancer. NEngl J Med. 2011;364(19): 1817-1825.

26. von Hoff DD, Ervin T, Arena FP, et al. Increased survival in pancreatic cancer with nab-paclitaxel plus gemcitabine. $N$ Engl J Med. 2013;369(18):1691-1703

27. Attard CL, Brown S, Alloul K, Moore MJ. Cost-effectiveness of folfirinox for first-line treatment of metastatic pancreatic cancer. Curr Oncol. 2014;21(1):41-51.

28. Uesaka K, Boku N, Fukutomi A, et al. Adjuvant chemotherapy of S-1 versus gemcitabine for resected pancreatic cancer: a phase 3, open-label, randomised, non-inferiority trial (JASPAC 01). Lancet. 2016;388(10041):248-257.

29. Zhou J, Zhao R, Wen F, et al. Cost-effectiveness analysis of treatments for metastatic pancreatic cancer based on PRODIGE and MPACT trials. Tumori. 2016;2016(3):294-300.

30. Murray CJ, Evans DB, Acharya A, Baltussen RM. Development of WHO guidelines on generalized cost-effectiveness analysis. Health Econ. 2000;9(3):235-251.

31. Wu B, Ye M, Chen H, Shen JF. Costs of trastuzumab in combination with chemotherapy for HER2-positive advanced gastric or gastroesophageal junction cancer: an economic evaluation in the Chinese context. Clin Ther. 2012;34(2):468-479.

32. Lazzaro C, Barone C, Caprioni F, et al. An Italian cost-effectiveness analysis of paclitaxel albumin (nab-paclitaxel) + gemcitabine vs gemcitabine alone for metastatic pancreatic cancer patients: the APICE study. Expert Rev Pharmacoecon Outcomes Res. 2018;18(4):435-446.

33. Coyle D, Ko YJ, Coyle K, et al. Cost-Effectiveness Analysis of Systemic Therapies in Advanced Pancreatic Cancer in the Canadian Health Care System. Value Health. 2017;20(4):586-592.

34. Zhou J, Zhao R, Wen F, et al. Cost-effectiveness analysis of gemcitabine, S-1 and gemcitabine plus S-1 for treatment of advanced pancreatic cancer based on GEST study. Med Oncol. 2015;32(4):121.

35. Lee SG, Jee YG, Chung HC, et al. Cost-effectiveness analysis of adjuvant therapy for node positive breast cancer in Korea: docetaxel, doxorubicin and cyclophosphamide (TAC) versus fluorouracil, doxorubicin and cyclophosphamide (FAC). Breast Cancer Res Treat. 2009; 114(3):589-595.

36. Liubao P, Xiaomin W, Chongqing T, et al. Cost-effectiveness analysis of adjuvant therapy for operable breast cancer from a Chinese perspective: doxorubicin plus cyclophosphamide versus docetaxel plus cyclophosphamide. Pharmacoeconomics. 2009;27(10):873-886. 


\section{Publish your work in this journal}

Cancer Management and Research is an international, peer-reviewed open access journal focusing on cancer research and the optimal use of preventative and integrated treatment interventions to achieve improved outcomes, enhanced survival and quality of life for the cancer patient. The manuscript management system is completely online and includes a very quick and fair peer-review system, which is all easy to use. Visit http://www.dovepress.com/testimonials.php to read real quotes from published authors.

Submit your manuscript here: https://www.dovepress.com/cancer-management-and-research-journal 